TRANSACTIONS OF THE

AMERICAN MATHEMATICAL SOCIETY

Volume 363, Number 9, September 2011, Pages 4777-4799

S 0002-9947(2011)05292-X

Article electronically published on March 25, 2011

\title{
GLOBAL SOLUTION BRANCH AND MORSE INDEX ESTIMATES OF A SEMILINEAR ELLIPTIC EQUATION WITH SUPER-CRITICAL EXPONENT
}

\author{
ZONGMING GUO AND JUNCHENG WEI
}

Abstract. We consider the nonlinear eigenvalue problem

$$
\left\{\begin{array}{l}
-\Delta u=u^{p}+\lambda u \text { in } B, \\
u>0 \text { in } B, \quad u=0 \text { on } \partial B,
\end{array}\right.
$$

where $B$ denotes the unit ball in $\mathbb{R}^{N}, N \geq 3, \lambda>0$ and $p>(N+2) /(N-2)$. According to classical bifurcation theory, the point $\left(\mu_{1}, 0\right)$ is a bifurcation point from which emanates an unbounded branch $\mathscr{C}$ of solutions $(\lambda, u)$ of $(0.1)$, where $\mu_{1}$ is the principal eigenvalue of $-\Delta$ in $B$ with Dirichlet boundary data. It is known that there is a unique value $\lambda=\lambda_{*} \in\left(0, \mu_{1}\right)$ such that $(0.1)$ has a radial singular solution $u_{*}(|x|)$. Let $p_{c}>\frac{N+2}{N-2}$ be the Joseph-Lundgren exponent. We show that the structure of the branch $\mathscr{C}$ changes for $p \geq p_{c}$ and $(N+2) /(N-2)<p<p_{c}$. For $(N+2) /(N-2)<p<p_{c}, \mathscr{C}$ turns infinitely many times around $\lambda_{*}$, which implies that all the singular solutions have infinite Morse index. For $p \geq p_{c}$, we show that all solutions (regular or singular) have finite Morse index. For $N \geq 12$ and $p>p_{c}$ large, we show that all solutions (regular or singular) have exactly Morse index one. As a consequence, we prove that any regular solution intersects with the singular solution exactly once and any regular solution exists (and is unique) only when $\lambda \in\left(\lambda_{*}, \mu_{1}\right)$.

\section{INTRODUCTION}

Let $B$ be the unit ball in $\mathbb{R}^{N}(N \geq 3)$. In this paper, we consider the following nonlinear eigenvalue problem:

$$
\left\{\begin{array}{l}
-\Delta u=u^{p}+\lambda u \text { in } B, \\
u>0 \text { in } B, \quad u=0 \text { on } \partial B
\end{array}\right.
$$

where

$$
p>p_{N}:=\frac{N+2}{N-2}, \lambda \in \mathbb{R} .
$$

By [8, any solution of problem (1.1) is radially symmetric. It is easy to see that there exist no solutions for (1.1) if $\lambda \geq \mu_{1}$ or $\lambda \leq 0$, where $\mu_{1}$ is the principal eigenvalue of $-\Delta$ in $B$ with Dirichlet data. According to classical bifurcation theory [15, the point $\left(\mu_{1}, 0\right)$ is a bifurcation point from which emanates an unbounded branch $\mathscr{C}$ of solutions $(\lambda, u)$. In this paper we are interested in the structure of the branch $\mathscr{C}$.

Received by the editors March 31, 2009 and, in revised form, November 5, 2009.

2010 Mathematics Subject Classification. Primary 35B33; Secondary 35B32, 35J61.

Key words and phrases. Branch of positive radial solutions, infinitely many turning points, Morse index, semilinear elliptic problems with super-critical exponent, Bessel function. 
For $1<p<\frac{N+2}{N-2}$, there exists at most one solution of (1.1) when $\lambda<\mu_{1}$ ([17]). When $p=\frac{N+2}{N-2}$, Brezis and Nirenberg established that (1.1) is solvable for $\bar{\lambda}<\lambda<\mu_{1}$, where $\bar{\lambda}=0$ when $N \geq 4$ and $\bar{\lambda}=\frac{1}{4} \mu_{1}$ when $N=3$. The situation drastically changes as soon as $p>\frac{N+2}{N-2}$. Del Pino-Dolbeault-Musso [5] constructed bubble-tower solutions of (1.1) for a slightly supercritical exponent in dimension $N \geq 4$. Budd and Norbury [2] used formal asymptotics and numerical computations to derive some qualitative properties of the solution branch when $N=3, p>5$. They found that before reaching $\lambda=0$, the solution curve turns right and oscillates infinitely many times in the form of an exponentially damped sinusoidal along a line $\lambda=\lambda_{*}$. Merle and Peletier [11] proved that there is a unique value $\lambda=\lambda_{*}>0$ such that a singular solution $u_{*}$ exists for (1.1). Moreover,

$$
u_{*}(r)=A(p, N) r^{-2 /(p-1)}\left\{1-B(p, N) r^{2}+o\left(r^{2}\right)\right\} \text { as } r \rightarrow 0,
$$

where

$$
A(p, N)=\left[\frac{2}{p-1}\left(N-2-\frac{2}{p-1}\right)\right]^{1 /(p-1)}, \quad B(p, N)=4 \lambda_{*}\left(N-1-\frac{3}{p-1}\right)^{-1} .
$$

Merle-Peletier-Serrin [12] studied the asymptotic behavior of the positive solutions $\left(\lambda_{p}, u_{p}\right) \in \mathscr{C}$ as $p \rightarrow \infty$. Recently, using the geometric theory of dynamical systems, Dolbeault and Flores [6] rigorously proved the numerical computations in [2] in the case of $p<p_{c}$ - the Joseph-Lungren exponent (see (1.5)).

An analogous problem

$$
\left\{\begin{array}{l}
-\Delta u=\lambda(1+u)^{p} \text { in } B \\
u>0 \text { in } B, \quad u=0 \text { on } \partial B
\end{array}\right.
$$

has been completely understood. It is known [10] that there exists a unique $\lambda_{*}>0$ such that the solution to (1.4) exists only when $\lambda \leq \lambda_{*}$. Let

$$
p_{c}:= \begin{cases}\infty & \text { if } 2 \leq N \leq 10 \\ \frac{(N-2)^{2}-4 N+8 \sqrt{N-1}}{(N-2)(N-10)} & \text { if } N \geq 11\end{cases}
$$

be the so-called Joseph-Lundgren exponent introduced in [10]. When $p<p_{c}$, there exists another number $\lambda^{*}<\lambda_{*}$ such that for $\lambda \in\left(\lambda^{*}, \lambda_{*}\right]$ the solution $u_{\lambda}$ exists and is smooth up to $\lambda=\lambda_{*}$, the singular solution exists at $\lambda=\lambda^{*}$, and the solution's branch turns infinitely many times at $\lambda=\lambda^{*}$. For $p \geq p_{c}$, there are no secondary branches and the singular solution exists precisely at $\lambda=\lambda_{*}$ and is stable.

The exponent $p_{c}$ has long been known to play an important role in semilinear heat equations with power-like nonlinearities. See Gui-Ni-Wang 9, Polacik-Yanagida [13, Fila-Winkler-Yanagida 7] and Wang [16] and the references therein.

The study of (1.1) turns out to be more difficult and delicate than (1.4). The main difficulty is that any solution (regular or singular) to (1.1) is unstable. An important question is then to estimate the Morse index of the solutions (regular or singular). In this paper we shall see that $p_{c}$ also plays an important role in the structure of the branch $\mathscr{C}$; i.e., $\mathscr{C}$ turns infinitely many times around $\lambda=\lambda_{*}$ provided $(N+2) /(N-2)<p<p_{c}$, but this does not occur when $p \geq p_{c}$. The first conclusion was obtained recently in [6] by using tools of the geometric theory of dynamical systems. Nothing is known for the case $p \geq p_{c}$. The results obtained in this paper for the case $p \geq p_{c}$ are new. In some cases, we obtain optimal results. We will use different methods to deal with this problem. We show that the Morse 
index of $u_{*}$ is $\infty$ provided $(N+2) /(N-2)<p<p_{c}$, but it is finite when $p \geq p_{c}$. To show the first conclusion, we use some arguments similar to those in [4]. We also present some sufficient conditions to guarantee that the Morse index of $u_{*}$ is 1 when $p \geq p_{c}$. Note that this holds only for $N \geq 11$ and is optimal.

The main results of this paper are summarized in the following three theorems:

Theorem 1.1. Assume $N \geq 3,(N+2) /(N-2)<p<p_{c}$. There is a unique number $\lambda_{*}>0$ such that, given any integer $k \geq 1$, there exist at least $k$ bounded radial solutions of (1.1) for any $\lambda$ sufficiently close to $\lambda_{*}$. In particular, there are infinitely many classical solutions of (1.1) for $\lambda=\lambda_{*}$.

Theorem 1.2. For $N \geq 11$ and $p \geq p_{c}$, the Morse index of any solution (singular or regular) $u_{\lambda}$ of (1.1) is finite. The graph of any regular solution intersects with that of the singular solution at most finitely many times.

Theorem 1.3. For $N \geq 12$ and $p \geq p_{c}^{2}(N)$, where $p_{c}^{2}(N) \geq p_{c}$ can be computed explicitly, the Morse index of any solution (singular or regular) $u_{\lambda}$ of (1.1) is exactly 1. The regular solution intersects with the singular solution only once, and any the regular solution exists and is unique when $\lambda \in\left(\lambda_{*}, \mu_{1}\right)$.

Theorem [1.1 has been proved by Dolbeault and Flores [6] using the geometric dynamical system method. Here we shall give a PDE proof. Theorems 1.2 and 1.3 are new. Theorem 1.3 gives a complete description of the solution branch. The existence of another supercritical exponent $p_{c}^{2} \geq p_{c}$ is interesting.

A simple estimate on the number $p_{c}^{2}(N)$ can be given as follows. Let $J_{\nu}(r)$ be the Bessel function satisfying

$$
J_{\nu}^{\prime \prime}+\frac{1}{r} J_{\nu}^{\prime}+\left(1-\frac{\nu^{2}}{r^{2}}\right) J_{\nu}=0, \quad r \in(0, \infty)
$$

Let $j_{1, \nu}$ and $j_{2, \nu}$ be the first two zeroes of $J_{\nu}(r)$. Then Theorem 1.3 holds under the following condition:

$$
j_{1, \frac{N-2}{2}} \leq j_{2, \nu}
$$

where

$$
\nu=\frac{1}{2} \sqrt{\left(N-2-\frac{4}{p-1}\right)^{2}-8\left(N-2-\frac{2}{p-1}\right)} \geq 0 \text { when } p \geq p_{c} .
$$

Notice that $\mu_{1}=j_{1, \frac{N-2}{2}}^{2}$. We also note that as $p \rightarrow+\infty$, condition (1.7) becomes

$$
j_{1, \frac{N-2}{2}} \leq j_{2, \frac{\sqrt{(N-2)(N-10)}}{2}}
$$

which can be shown to hold when $N \geq 12$. This is also the reason behind the condition $N \geq 12$ in Theorem 1.3 .

Finally, let us also observe the following fact:

$$
\begin{array}{ll}
\frac{2 p}{p-1}\left(N-2-\frac{2}{p-1}\right)>\frac{(N-2)^{2}}{4} & \text { for } \frac{N+2}{N-2}<p<p_{c} \\
\frac{2 p}{p-1}\left(N-2-\frac{2}{p-1}\right) \leq \frac{(N-2)^{2}}{4} & \text { for } p \geq p_{c} .
\end{array}
$$


This also implies

$$
\begin{array}{ll}
\left(N-2-\frac{4}{p-1}\right)^{2}-8\left(N-2-\frac{2}{p-1}\right)<0 & \text { for } \frac{N+2}{N-2}<p<p_{c} \\
\left(N-2-\frac{4}{p-1}\right)^{2}-8\left(N-2-\frac{2}{p-1}\right)>0 & \text { for } p>p_{c} \\
\left(N-2-\frac{4}{p-1}\right)^{2}-8\left(N-2-\frac{2}{p-1}\right)=0 & \text { for } p=p_{c}
\end{array}
$$

The organization of the paper is as follows: in Section 2, we prove Theorem 1.1. In Section 3, we show that the Morse index of any solution is finite, and then we prove Theorem 1.2. In Section 4, we prove that under some conditions the Morse index of any solution is one, and thus prove Theorem 1.3. We leave the proof of one key theorem to Section 5. In Section 6, we present some estimates on the exponent $p_{c}^{2}$.

\section{The Case for $(N+2) /(N-2)<p<p_{c}$ : Proof of Theorem 1.1}

In this section we present a PDE proof of Theorem 1.1. Throughout this section, we assume that $\frac{N+2}{N-2}<p<p_{c}$. We only need to consider the equation

$$
\left\{\begin{array}{l}
-u^{\prime \prime}-\frac{N-1}{r} u^{\prime}=u^{p}+\lambda u \text { in }(0,1) \\
u>0 \operatorname{in}^{(0,1),} u(1)=0
\end{array}\right.
$$

Let $w=\lambda^{-1 /(p-1)} u$. Then $w$ satisfies

$$
\left\{\begin{array}{l}
-w^{\prime \prime}-\frac{N-1}{r} w^{\prime}=\lambda\left[w^{p}+w\right] \text { in }(0,1) \\
w>0 \text { in }(0,1), w(1)=0
\end{array}\right.
$$

We also know that there is a unique $\lambda=\lambda_{*}$ such that (2.2) has a singular solution $w_{*}([1])$. Introducing the rescaling

$$
t=\lambda_{*}^{1 / 2} r, \quad W(t)=w_{*}(r),
$$

we see that $W$ satisfies

$$
\left\{\begin{array}{l}
-W^{\prime \prime}-\frac{N-1}{t} W^{\prime}=W^{p}+W \text { in }\left(0, \lambda_{*}^{1 / 2}\right) \\
W>0 \text { in }\left(0, \lambda_{*}^{1 / 2}\right), W\left(\lambda_{*}^{1 / 2}\right)=0
\end{array}\right.
$$

Moreover, we can choose subsequences $\left.\left\{\left(\lambda_{n}, u_{n}\right)\right\} \equiv\left(\lambda_{n}, u_{\lambda_{n}}\right)\right\} \subset \mathscr{C}$ with $\lambda_{n} \rightarrow \lambda_{*}$, $\max _{B} u_{n} \rightarrow \infty$ as $n \rightarrow \infty$. Then by the changes

$$
t=\lambda_{n}^{1 / 2} r, \quad W_{n}(t)=\lambda_{n}^{-1 /(p-1)} u_{n}(r)
$$

we see that $W_{n}$ satisfies the problem:

$$
\left\{\begin{array}{l}
-W_{n}^{\prime \prime}-\frac{N-1}{t} W_{n}^{\prime}=W_{n}^{p}+W_{n} \text { in }\left(0, \lambda_{n}^{1 / 2}\right), \\
W_{n}>0 \text { in }\left(0, \lambda_{n}^{1 / 2}\right), \quad W_{n}\left(\lambda_{n}^{1 / 2}\right)=0 .
\end{array}\right.
$$

It is known from Theorem 1.2 of [1] that $W_{n} \rightarrow W$ in $C_{l o c}^{0}\left(0, \lambda_{*}^{1 / 2}\right)$ as $n \rightarrow \infty$.

We have the following proposition.

Proposition 2.1. For any sequence $\left\{\left(\lambda_{n}, W_{n}\right)\right\} \equiv\left\{\left(\lambda_{n}, W_{\lambda_{n}}\right)\right\}$ with $\lambda_{n} \rightarrow \lambda_{*}$, $\max _{B} W_{n} \rightarrow \infty$ and any $M \gg 1$, there is $N^{*}=N^{*}(M)>1$ such that for $n>N^{*}$, the graph of $W_{n}$ intersects with that of $W$ at least $M$ times in $\left(0, \min \left\{\lambda_{n}^{1 / 2}, \lambda_{*}^{1 / 2}\right\}\right)$. 
Proposition 2.1 implies that the Morse index of $W$ is $\infty$ and hence the Morse index of $u_{*}$ is $\infty$. Indeed, we see that $W-W_{n}$ has at least $M$ zeroes in $\left(0, \min \left\{\lambda_{n}^{1 / 2}, \lambda_{*}^{1 / 2}\right\}\right)$, and thus there are at least $\left[\frac{M}{2}\right]-1$ intervals $I_{i}\left(i=1,2, \ldots,\left[\frac{M}{2}\right]-\right.$ 1) on which $W-W_{n}>0$. We also see that $h_{n}^{i}:=W-W_{n}$ satisfies

$$
\left\{\begin{array}{l}
-\Delta h_{n}^{i}<p W^{p-1} h_{n}^{i}+h_{n}^{i} \text { in } I_{i}, \\
h_{n}^{i}>0 \text { in } I_{i}, \quad h_{n}^{i}=0 \text { on } \partial I_{i} .
\end{array}\right.
$$

Multiplying $h_{n}^{i}$ on both sides of the equation in (2.5) and integrating it on the annular domain $\Omega_{i}:=\left\{y:|y| \in I_{i}\right\}$, we then obtain

$$
\int_{\Omega_{i}}\left[\left|\nabla h_{n}^{i}\right|^{2}-\left(p W^{p-1}+1\right)\left(h_{n}^{i}\right)^{2}\right]<0 .
$$

Since for each $h_{n}^{i} \in H_{0}^{1}\left(B_{*}\right)$, where $B_{*}:=\left\{y:|y|<\lambda_{*}^{1 / 2}\right\}$,

$$
\int_{B_{*}} h_{n}^{i} h_{n}^{j} d y=0, \quad i \neq j,
$$

the arbitrariness of $M$ implies that the Morse index of $W$ is $\infty$.

Proof of Proposition 2.1. The existence of $\left\{\left(\lambda_{n}, W_{n}\right)\right\}$ is known from the existence of the sequence $\left\{\left(\lambda_{n}, u_{n}\right)\right\}$. On the contrary, this is a sequence $\left\{\left(\lambda_{n}, W_{n}\right)\right\}$ satisfying the conditions in this proposition, so we have that there is $t_{0} \in\left(0, \min \left\{\lambda_{n}^{1 / 2}, \lambda_{*}^{1 / 2}\right\}\right)$ independent of $n$ such that $W>W_{n}$ in $\left(0, t_{0}\right)$ (note that $W(0)=\infty$ ). Since $W_{n}(t)=\lambda_{n}^{-1 /(p-1)} u_{n}$, it follows from Theorem 1.2 of [1] that

$$
W_{n} \rightarrow W \text { in } C_{l o c}^{0}\left(0, \lambda_{*}^{1 / 2}\right) \text { as } n \rightarrow \infty .
$$

Let $z_{n}(\rho)=W_{n}(t) / W(t), \rho=\ln t$. Then $z_{n}$ satisfies

$$
z_{n}^{\prime \prime}(\rho)+\left[N-2+\frac{2 t W_{t}(t)}{W}\right] z_{n}^{\prime}(\rho)+t^{2} W^{p-1}\left[z_{n}^{p}-z_{n}\right]=0, \quad \rho<\ln t_{0} .
$$

Since $w_{*}(r)=\lambda_{*}^{-1 /(p-1)} u_{*}(r)$ and $W(t)=w_{*}(r)$ with $t=\lambda_{*}^{1 / 2} r$, we see from (1.2) that

$$
W(t)=A(p, N) t^{-2 /(p-1)}\left(1-B(p, N) \lambda_{*}^{-1} t^{2}+o\left(t^{2}\right)\right) \text { as } t \rightarrow 0
$$

Thus,

$$
\begin{gathered}
\frac{2 t W_{t}(t)}{W(t)} \leq-\frac{4}{p-1} \text { for } t \text { sufficiently small, } \\
\frac{2 t W_{t}(t)}{W(t)} \rightarrow-\frac{4}{p-1} \text { as } t \rightarrow 0
\end{gathered}
$$

and

$$
\begin{gathered}
t^{2} W^{p-1}(t) \leq \frac{2}{p-1}\left(N-2-\frac{2}{p-1}\right) \text { for } t \text { sufficiently small, } \\
t^{2} W^{p-1}(t) \rightarrow \frac{2}{p-1}\left(N-2-\frac{2}{p-1}\right) \text { as } t \rightarrow 0
\end{gathered}
$$

Since $z_{n}(\rho)<1$ for $\rho \in\left(-\infty, \ln t_{0}\right)$ and $W(t) \rightarrow \infty$ as $t \rightarrow 0$, we conclude that there exists $-\infty<T<\ln t_{0}$ such that

$$
z_{n}^{\prime \prime}(\rho)+g_{1}(\rho) z_{n}^{\prime}(\rho) \geq 0 \text { for } \rho \in(-\infty, T),
$$


where $g_{1}(\rho)=N-2+\frac{2 t W_{t}(t)}{W(t)}$. Thus,

$$
\exp \left(\int_{-\infty}^{\rho} g_{1}(s) d s\right) z_{n}^{\prime}(\rho) \geq \exp \left(\int_{-\infty}^{\tau} g_{1}(s) d s\right) z_{n}^{\prime}(\tau) \text { if } t \geq \tau>-\infty .
$$

We know that $g_{1}(\rho) \rightarrow N-2-\frac{4}{p-1}$ as $\rho \rightarrow-\infty$ and $z_{n}(\rho) \rightarrow 0$ as $\rho \rightarrow-\infty$. Then it follows from (2.13) that

$$
z_{n}^{\prime}(\rho) \geq 0 \text { for }-\infty<\rho<T .
$$

Thus, $z_{n}^{\prime}(\rho) \not \equiv 0$ since $W_{n} \not \equiv W$.

Let $\omega_{n}(\rho)=1-z_{n}(\rho)$. Then by (2.6) , we have

$$
\omega_{n}^{\prime \prime}(\rho)+g_{1}(\rho) \omega_{n}^{\prime}+g_{2}^{n}(\rho) \omega_{n}=0, \omega_{n}>0 \text { on }(-\infty, T),
$$

where

$$
g_{2}^{n}(\rho)=t^{2} W^{p-1}(t)\left[\frac{z_{n}^{p}-z_{n}}{1-z_{n}}\right] .
$$

Since $z_{n} \rightarrow 1$ in $C_{l o c}^{0}(-\infty, T)$ as $n \rightarrow \infty$, we obtain that

$$
\frac{z_{n}^{p}-z_{n}}{1-z_{n}} \rightarrow-(p-1) \text { in } C_{l o c}^{0}(-\infty, T) \text { as } n \rightarrow \infty .
$$

This, together with the fact that

$$
t^{2} W^{p-1}(t) \rightarrow \frac{2}{p-1}\left(N-2-\frac{2}{p-1}\right) \text { as } t \rightarrow 0
$$

and

$$
\left(N-2-\frac{4}{p-1}\right)^{2}-8\left(N-2-\frac{2}{p-1}\right)<0 \text { for }(N+2) /(N-2)<p<p_{c},
$$

imply that there exists $T^{*} \in(-\infty, T)$ such that for any interval $\left[T_{2}, T_{1}\right] \subset\left(-\infty, T^{*}\right)$ and $n$ sufficiently large,

$$
\left[g_{1}\right]^{2}-4 g_{2}^{n}<0 \text { in }\left[T_{2}, T_{1}\right] .
$$

Thus there exist $b_{1}$ and $c_{1}$ such that $b_{1}^{2}-4 c_{1}<0, g_{1}(\rho)<b_{1}$ and $g_{2}^{n}(\rho)>c_{1}$ if $\rho \in\left[T_{2}, T_{1}\right]$. Observe that any solution of

$$
Z^{\prime \prime}(\rho)+b_{1} Z^{\prime}(\rho)+c_{1} Z(\rho)=0
$$

is oscillatory. In particular, there exist $T_{2}<a_{2}<b_{2}<T_{1}$ such that $Z\left(a_{2}\right)=$ $Z\left(b_{2}\right)=0, Z>0$ in $\left(a_{2}, b_{2}\right)$ (and hence $Z^{\prime}\left(a_{2}\right)>0>Z^{\prime}\left(b_{2}\right)$ ). Multiplying (2.15) by $Z$ and (2.17) by $\omega_{n}$, we have

$$
\begin{gathered}
\omega_{n}^{\prime \prime} Z+g_{1}(\rho) \omega_{n}^{\prime} Z+g_{2}^{n}(\rho) \omega_{n} Z=0 \text { on }\left[a_{2}, b_{2}\right], \\
Z^{\prime \prime} \omega_{n}+b_{1} Z^{\prime} \omega_{n}+c_{1} Z \omega_{n}=0 \text { on }\left[a_{2}, b_{2}\right] .
\end{gathered}
$$

Subtracting (2.19) from (2.18) yields

$$
\left(Z \omega_{n}^{\prime}-Z^{\prime} \omega_{n}\right)^{\prime}+\left(g_{1}(\rho) \omega_{n}^{\prime} Z-b_{1} Z^{\prime} \omega_{n}\right)+\left(g_{2}^{n}-c_{1}\right) \omega_{n} Z=0 \text { on }\left[a_{2}, b_{2}\right] .
$$

Thus, by the fact that $g_{1}(\rho)<b_{1}, g_{2}^{n}(\rho)>c_{1}$ and $\omega_{n}^{\prime} \leq 0$, we have

$$
\left(Z \omega_{n}^{\prime}-Z^{\prime} \omega_{n}\right)^{\prime}+b_{1}\left(\omega_{n}^{\prime} Z-Z^{\prime} \omega_{n}\right)<0 \text { on }\left(a_{2}, b_{2}\right)
$$

and hence

$$
e^{b_{1} b_{2}} Z^{\prime}\left(b_{2}\right) \omega_{n}\left(b_{2}\right)>e^{b_{1} a_{2}} Z^{\prime}\left(a_{2}\right) \omega_{n}\left(a_{2}\right) .
$$

This is impossible (note that $Z^{\prime}\left(a_{2}\right)>0>Z^{\prime}\left(b_{2}\right)$ ) and completes the proof. 
To prove Theorem 1.1, we only need to obtain similar results for the problem (2.2). According to classical theory [15], the point $\left(\mu_{1}, 0\right)$ is a bifurcation point from which emanates an unbounded branch $\mathscr{A}$ of solutions $(\lambda, w)$ of (2.2).

We prove the following theorem.

Theorem 2.2. Let $N \geq 3$ and $\frac{N+2}{N-2}<p<p_{c}$. Then the radial solution branch $\mathscr{A}$ of (2.2) possesses infinitely many turning points around $\lambda=\lambda_{*}$.

It is clear that Theorem 2.2 implies that the conclusions of Theorem 1.1 hold. To prove Theorem 2.2, we first prove the following lemma, which implies that the radial solution branch $\mathscr{A}$ of (2.2) has no secondary bifurcation point.

Lemma 2.3. For any $\kappa \in(0, \infty)$, there is at most one $\tilde{\lambda}:=\tilde{\lambda}(\kappa) \in\left(0, \mu_{1}\right)$ with $\left(\tilde{\lambda}, w_{\tilde{\lambda}}\right) \in \mathscr{A}$ and $w_{\tilde{\lambda}}(0)=\kappa$.

Proof. Suppose that there are $\lambda_{1}, \lambda_{2} \in\left(0, \mu_{1}\right)$ with $\lambda_{1} \neq \lambda_{2}$ and $\left(\lambda_{1}, w_{\lambda_{1}}\right),\left(\lambda_{2}, w_{\lambda_{2}}\right)$ $\in \mathscr{A}$ such that $w_{\lambda_{1}}(0)=w_{\lambda_{2}}(0)=\kappa$. If we set $w_{j} \equiv w_{\lambda_{j}}$ for $j=1,2$, then

$$
-w_{j}^{\prime \prime}-\frac{N-1}{r} w_{j}^{\prime}=\lambda_{j}\left[w_{j}^{p}+w_{j}\right], w_{j}(0)=\kappa, w_{j}^{\prime}(0)=0, \quad w_{j}(1)=0 .
$$

Let $t=\lambda_{j}^{1 / 2} r$ and $z_{j}(t)=w_{j}(r)$. Then $z_{j}$ satisfies

$$
-z_{j}^{\prime \prime}-\frac{N-1}{t} z_{j}^{\prime}=z_{j}^{p}+z_{j}, \quad z_{j}(0)=\kappa, \quad z_{j}^{\prime}(0)=0, \quad z_{j}\left(\lambda_{j}^{1 / 2}\right)=0 .
$$

Since $z_{1}$ and $z_{2}$ satisfy the same initial values $z_{j}(0)=\kappa, z_{j}^{\prime}(0)=0$, the standard ODE theory implies $\lambda_{1}=\lambda_{2}$, which is a contradiction. This completes the proof.

Lemma 2.3 implies that the radial solution branch $\mathscr{A}$ of (2.2) does not possess a secondary bifurcation point.

Proof of Theorem 2.2, The proof of Theorem 2.2 can be obtained from Proposition 2.1 and arguments similar to those in [4].

Arguments similar to those in the proof of Proposition 2.1 imply that for any $M \gg 1$, we can find $N^{*}=N^{*}(M)$ such that for $n>N^{*}$, the Morse index of $W_{n}$ is at least $\left[\frac{M}{2}\right]-1$. (Note that instead of choosing $h_{n}^{i}=W-W_{n}$, we can choose $h_{n}^{i}=W_{n}-W$.) We can argue as in Subsection 2.1 of [3], in the space $C^{1}([0,1]) \times \mathbb{R}$, to find an analytic solution curve of (2.2): $\lambda=\tilde{\lambda}(s), w=\tilde{w}(s)$ for $s \geq 0$, such that

$$
\begin{aligned}
& \|\tilde{w}\|_{\infty} \rightarrow \infty \text { as } s \rightarrow \infty \\
& (\tilde{w}(s), \tilde{\lambda}(s)) \in \mathscr{A} \text { for } s \geq 0 \\
& (\tilde{w}(0), \tilde{\lambda}(0))=\left(0, \mu_{1}\right) \text { and } \\
& I-\tilde{\lambda}(s) A^{\prime}(\tilde{w}(s)) \text { is invertible except at isolated points, }
\end{aligned}
$$

where $A^{\prime}(\tilde{w}(s)):=G^{-1}\left[\left(p \tilde{w}^{p-1}(s)+1\right) I\right]$ and $G(h)=r^{1-N}\left(r^{N-1} h^{\prime}(r)\right)^{\prime}$ with $h^{\prime}(0)=$ $0, h(1)=0$. We see from Lemma 2.3 that the curve has no self-intersection. Let us denote this curve by $T$ and let $\mu_{i, \tilde{\lambda}(s)}(\tilde{w}(s))$ be the $i$ th eigenvalue, counting the multiplicity, of

$$
-G-\tilde{\lambda}(s)\left[p \tilde{w}^{p-1}(s)+1\right] I
$$

on $(0,1)$ with the Dirichlet boundary condition. By our comments above, $\mu_{i, \tilde{\lambda}(s)}(\tilde{w}(s))$ are continuous and piecewise analytic, and have only isolated zeroes. We will show that $\mu_{i, \tilde{\lambda}(s)}(\tilde{w}(s))<0$ for $s$ large. This means that for any $\zeta>0$, 
equation (2.22) has at least $\zeta$ negative eigenvalues for $s$ large. Hence we conclude that there is a sequence $\left\{s_{i}\right\}$ with $s_{i} \rightarrow \infty$ as $i \rightarrow \infty$ such that the number of negative eigenvalues of (2.22) (counting multiplicity) changes at $s_{i}$. (Recall that $\mu_{i, \tilde{\lambda}(0)}(\tilde{w}(0))=\mu_{i}(-G)-\mu_{1} \rightarrow+\infty$ as $i \rightarrow \infty$.) Each $\left(\tilde{w}\left(s_{i}\right), \tilde{\lambda}\left(s_{i}\right)\right)$ must be a bifurcation point. We also know that each $\left(\tilde{w}\left(s_{i}\right), \tilde{\lambda}\left(s_{i}\right)\right)$ is either a turning point or a point of secondary bifurcation. Our Lemma 2.3 implies that it is not a secondary bifurcation point. Thus, it must be a turning point.

To prove our claim on $\mu_{i, \tilde{\lambda}(s)}$ for large $s$, we need to consider positive solutions $\left(w_{i}, \lambda_{i}\right)$ of (2.2) such that $\lambda_{i} \rightarrow \lambda_{*}$ as $i \rightarrow \infty$ and $\left\|w_{i}\right\|_{\infty} \rightarrow \infty$ as $i \rightarrow \infty$. Thus, there is an $s_{i}$ with $s_{i} \rightarrow \infty$ such that $\tilde{\lambda}\left(s_{i}\right)=\lambda_{i}$ and $\tilde{w}\left(s_{i}\right)=w_{i}$. By the changes

$$
z_{i}(\rho)=w_{i}(r), \quad \rho=\lambda_{i}^{1 / 2} r
$$

we see that $z_{i}$ satisfies

$$
-z_{i}^{\prime \prime}-\frac{N-1}{\rho} z_{i}^{\prime}=z_{i}^{p}+z_{i} \text { in }\left(0, \lambda_{i}^{1 / 2}\right), \quad z_{i}\left(\lambda_{i}^{1 / 2}\right)=0 .
$$

It is known from Proposition 2.1 that for any $M \gg 1$, the Morse index of $z_{i}$ in $H_{0}^{1}\left(\tilde{B}_{i}\right)$, where $\tilde{B}_{i}=\left\{y:|y|<\lambda_{i}^{1 / 2}\right\}$, is larger than $M$ provided $i$ is sufficiently large. Hence, returning to the original scaling we obtain that there is at least an $(M-1)$-dimensional subspace $E_{i}$ of $H_{0}^{1}(B)$ such that

$$
\int_{B}\left[|\nabla h|^{2}-\tilde{\lambda}(s)\left(p \tilde{w}^{p-1}(s)+1\right) h^{2}\right]<0
$$

for $h$ in the unit sphere of $E_{i}$ and $s$ large. By the variational characterization of eigenvalues, this implies that $\mu_{i, \tilde{\lambda}(s)}(\tilde{w}(s))<0$ for $1 \leq i \leq M-1$ if $s$ is large. Since $M$ is arbitrary, this proves our claim and completes the proof of Theorem 2.2 .

\section{The Case For $p \geq p_{c}$ : Proof of Theorem 1.2}

In this section we will study the structure of the radial solution branch $\mathscr{C}$ for $p \geq p_{c}$. We first show that for any $\left(\lambda, u_{\lambda}\right) \in \mathscr{C}$, the Morse index of $u_{\lambda}$ is bounded. Then we prove Theorem 1.2 .

We state our first result.

Theorem 3.1. There exists an integer $C \geq 1$ independent of $\lambda$ such that

$$
1 \leq m\left(u_{\lambda}\right) \leq C .
$$

To prove Theorem 3.1, we need the following key estimate.

Lemma 3.2. Let $u_{\lambda}$ be a regular or singular solution of (1.1). Then it holds that

$$
u_{\lambda}(r) \leq A(p, N) r^{-\frac{2}{p-1}} \text { for } r \in[0,1]
$$

where $A(p, N)$ is given in (1.3).

Proof. We first prove that (3.2) holds for a regular solution $u_{\lambda}$. To prove (3.2), we introduce the Emden-Fowler transformation for $u_{\lambda}$ :

$$
v_{\lambda}(t)=r^{\frac{2}{p-1}} u_{\lambda}(r), \quad t=\ln r .
$$

Then $v_{\lambda}$ satisfies the following problem (without loss of generality, we omit the subscript $\lambda$ on $v_{\lambda}$ ):

$$
v^{\prime \prime}+\alpha v^{\prime}+v^{p}-\beta v+\lambda e^{2 t} v=0, \quad t \in(-\infty, 0), \quad v(0)=0, \quad v(-\infty)=0,
$$


where

$$
\alpha=N-2-\frac{4}{p-1}, \quad \beta=A(p, N)^{p-1} .
$$

Now we show that

$$
v(t) \leq \beta^{1 /(p-1)}(=A(p, N)) \text { for } t \in(-\infty, 0),
$$

which implies (3.2).

Suppose the contrary: since $v(-\infty)=0$, we see that there is a $-\infty<\tilde{t}<0$ such that $v(\tilde{t})=A(p, N)$ but $v(t)<A(p, N)$ for $t \in(-\infty, \tilde{t})$.

Let $v(t)=A(p, N)+\varphi$. Then $\varphi(t) \leq 0$ for $t \in(-\infty, \tilde{t}]$ and

$$
v^{p}(t) \geq A(p, N)^{p}+p A(p, N)^{p-1} \varphi \text { for } t \in(-\infty, \tilde{t}) .
$$

Hence

$$
\begin{aligned}
0 & =\varphi^{\prime \prime}+\alpha \varphi^{\prime}+(A(p, N)+\varphi)^{p}-\beta(A(p, N)+\varphi)+\lambda e^{2 t}(A(p, N)+\varphi) \\
& \geq \varphi^{\prime \prime}+\alpha \varphi^{\prime}+(p-1) \beta \varphi .
\end{aligned}
$$

Letting $\varphi(t)=e^{\tau_{1} t} \tilde{\varphi}(t)$, where

$$
\tau_{1}=-\frac{\alpha}{2}+\frac{1}{2} \sqrt{\alpha^{2}-4(p-1) \beta},
$$

we have that $\tilde{\varphi}$ satisfies

$$
\left\{\begin{array}{l}
\tilde{\varphi}^{\prime \prime}+\left(\alpha+2 \tau_{1}\right) \tilde{\varphi}^{\prime} \leq 0, \quad t \in(-\infty, \tilde{t}), \\
\tilde{\varphi}(\tilde{t})=0, \quad \tilde{\varphi}(-\infty)=0,
\end{array}\right.
$$

where we have used the fact that (see (1.10) $)$

$$
\alpha^{2}-4(p-1) \beta \geq 0 \text { for } p \geq p_{c}
$$

and

$$
\tau_{1}=-\frac{\alpha}{2}+\frac{1}{2} \sqrt{\alpha^{2}-4(p-1) \beta}<0 .
$$

This contradicts the maximum principle since $\tilde{\varphi} \leq 0$ in $(-\infty, \tilde{t})$. This contradiction implies that (3.2) holds.

To show that the estimate (3.2) holds for $u_{*}$, we observe that under the changes

$$
v_{*}(t)=r^{2 /(p-1)} u_{*}(r), \quad t=\ln r,
$$

$v_{*}$ satisfies

$$
v_{*}^{\prime \prime}+\alpha v_{*}^{\prime}-\beta v_{*}+v_{*}^{p}+\lambda_{*} e^{2 t} v_{*}=0 \quad t \in(-\infty, 0), \quad v_{*}(-\infty)=A(p, N), \quad v_{*}(0)=0 .
$$

However, (1.2) implies that

$$
v_{*}(t)=A(p, N)-A(p, N) B(p, N) e^{2 t}+o\left(e^{2 t}\right) \text { for } t \text { near }-\infty .
$$

Therefore, if we set $v_{*}=A(p, N)+\varphi$, we then have that $\varphi(t)=-A(p, N) B(p, N) e^{2 t}$ $+o\left(e^{2 t}\right)<0$ for $t$ near $-\infty$ and

$$
e^{-\tau_{1} t} \varphi(t) \rightarrow 0 \text { as } t \rightarrow-\infty
$$

The fact that

$$
v_{*}(t) \leq A(p, N) \text { for } t \in(-\infty, 0)
$$

can be obtained by arguments similar to those in the proof of (3.4).

This proves Lemma 3.2 .

Next we prove that the Morse index of $u_{\lambda}$ is finite. 
Proof of Theorem 3.1, By (3.2), (1.10) and Hardy's inequality, we have that

$$
\int_{B}\left[|\nabla \phi|^{2}-p u_{\lambda}^{p-1} \phi^{2}\right] \geq \int_{B}\left[|\nabla \phi|^{2}-\frac{(N-2)^{2}}{4} \frac{\phi^{2}}{r^{2}}\right] \geq 0
$$

for any $\phi \in H_{0}^{1}(B)$. On the other hand, $m\left(u_{\lambda}\right) \geq 1$, since $u_{\lambda} \in H_{0}^{1}(B)$, and

$$
\int_{B}\left[\left|\nabla u_{\lambda}\right|^{2}-\left(p u_{\lambda}^{p-1}+\lambda\right) u_{\lambda}^{2}\right]=(1-p) \int_{B} u_{\lambda}^{p+1}<0 .
$$

To show that the Morse index of $u_{\lambda}$ is finite, we use a contradiction argument. By contradiction, there would be a sequence $\left\{\left(\lambda_{n}, u_{n}\right)\right\} \equiv\left\{\left(\lambda_{n}, u_{\lambda_{n}}\right)\right\} \subset \mathscr{C}$ such that

$$
m\left(u_{n}\right) \rightarrow \infty \text { as } n \rightarrow \infty .
$$

We can choose a subsequence (still denoted by $\left.\left\{\left(\lambda_{n}, u_{n}\right)\right\}\right)$ such that $\lambda_{n} \rightarrow \hat{\lambda}$ as $n \rightarrow \infty$. Thus, for $n$ sufficiently large, the number of negative eigenvalues (counting their multiplicity) of the problem

$$
-\Delta \varphi=\left[p u_{n}^{p-1}+\lambda_{n}\right] \varphi+\sigma \varphi \text { in } B, \quad \varphi=0 \text { on } \partial B
$$

is large. Therefore, the first eigenvalue $\sigma_{1}^{n}$ of the problem (3.10) satisfies

$$
\sigma_{1}^{n}+\hat{\lambda}<0 \text {. }
$$

This is a contradiction since

$$
0 \leq \int_{B}\left[\left|\nabla \varphi_{1}^{n}\right|^{2}-p u_{n}^{p-1}\left(\varphi_{1}^{n}\right)^{2}\right]=\left(\sigma_{1}^{n}+\lambda_{n}\right) \int_{B}\left(\varphi_{1}^{n}\right)^{2}<0,
$$

where $\varphi_{1} \in H_{0}^{1}(B)$ is the first eigenfunction of (3.10) corresponding to $\sigma_{1}^{n}$.

This proves Theorem 3.1

Proof of Theorems 1.2. The first conclusion follows from Theorem 3.1. We only need to show that the graph of any regular solution $u_{\lambda}$ of (1.1) intersects with that of $u_{*}$ at most finitely many times.

On the contrary, there would be $\left(\lambda, u_{\lambda}\right)$ such that the graph of $u_{\lambda}$ intersects with that of $u_{*}$ infinitely many times. There are two cases here: $\lambda \geq \lambda_{*}$ and $\lambda<\lambda_{*}$. For $\lambda \geq \lambda_{*}$, we can show that $m\left(u_{*}\right)=\infty$. This contradicts Theorem 3.1. Indeed, since the graph of $u_{\lambda}$ intersects with that of $u_{*}$ infinitely many times, there are infinitely many intervals $J_{i} \subset(0,1)(i=1,2, \ldots)$ such that $u_{*}>u_{\lambda}$ in $J_{i}$. Let

$$
h_{i}= \begin{cases}u_{*}-u_{\lambda}, & \text { in } J_{i}, \\ 0, & \text { in }(0,1) \backslash J_{i} .\end{cases}
$$

Thus we have that

$$
\int_{B_{i}}\left[\left|\nabla h_{i}\right|^{2}-\left(p u_{*}^{p-1}+\lambda_{*}\right) h_{i}^{2}\right] d x<0
$$

provided $\lambda \geq \lambda_{*}$, where $B_{i}=\left\{x,|x| \in J_{i}\right\}$. Note that

$$
-\Delta h_{i}<p u_{*}^{p-1} h_{i}+\lambda_{*} h_{i} \text { in } J_{i} .
$$

This implies that $m\left(u_{*}\right)=\infty$.

For $\lambda<\lambda_{*}$, we can show that $m\left(u_{\lambda}\right)=\infty$. This contradicts Theorem 3.1 again. Indeed, similarly, there are infinitely many intervals $J_{k} \subset(0,1)(k=1,2, \ldots)$ such that $u_{\lambda}>u_{*}$ in $J_{k}$. Let

$$
h_{k}= \begin{cases}u_{\lambda}-u_{*}, & \text { in } J_{k}, \\ 0, & \text { in }(0,1) \backslash J_{k} .\end{cases}
$$


We see that

$$
\int_{B_{k}}\left[\left|\nabla h_{k}\right|^{2}-\left(p u_{\lambda}^{p-1}+\lambda\right) h_{k}^{2}\right] d x<0
$$

provided $\lambda<\lambda_{*}$, where $B_{k}=\left\{x,|x| \in J_{k}\right\}$. Note that

$$
-\Delta h_{k}<p u_{\lambda}^{p-1} h_{k}+\lambda h_{k} \text { in } J_{k} .
$$

This implies that $m\left(u_{\lambda}\right)=\infty$.

The proof above also implies that the graphs of any two different regular solutions can only intersect finitely many times. This completes the proof.

\section{Morse index one solutions And the proof of Theorem 1.3}

In this section, we show that under some conditions, the Morse index of any solution is exactly one, and thus we prove Theorem 1.3

To this end, it is vital to study the following linearized operator at the singular solution:

$$
-\Delta h=\left(p u_{*}^{p-1}+\lambda_{*}\right) h \text { in } B .
$$

First, we note that under the Emden-Fowler transformation,

$$
\psi(t)=r^{2 /(p-1)} h(r), \quad t=\ln r,
$$

$\psi(t)$ satisfies the equation

$$
\psi^{\prime \prime}+\alpha \psi^{\prime}-\beta \psi+p v_{*}^{p-1} \psi+\lambda_{*} e^{2 t} \psi=0 \quad t \in(-\infty, 0) .
$$

Since $v_{*}(t) \rightarrow A(p, N)$ as $t \rightarrow-\infty$ and the characteristic equation of

$$
\psi^{\prime \prime}+\alpha \psi^{\prime}+(p-1) \beta \psi=0
$$

is

$$
\xi^{2}+\alpha \xi+(p-1) \beta=0,
$$

(4.2) has two fundamental solutions $\psi_{1}(t)$ and $\psi_{2}(t)$ with

$$
\psi_{1}(t) \sim e^{\xi_{1} t}, \quad \psi_{2}(t) \sim e^{\xi_{2} t} \text { as } t \rightarrow-\infty,
$$

where

$$
\xi_{1}=-\frac{\alpha}{2}+\frac{1}{2} \sqrt{\alpha^{2}-4(p-1) \beta}, \quad \xi_{2}=-\frac{\alpha}{2}-\frac{1}{2} \sqrt{\alpha^{2}-4(p-1) \beta} .
$$

Note that

$$
\xi_{1}<0, \quad \xi_{2}<0, \quad\left|\xi_{2}\right|>\left|\xi_{1}\right|>2,
$$

provided that $p \geq p_{c}$.

Our main result on (4.1) is the following.

Theorem 4.1. There exists $P:=p_{c}^{2}(N)>0$ such that if $p \geq \max \left\{p_{c}, P, 2\right\}$, then $\psi_{1}(0)<0$. As a consequence, problem (4.1) has a weak radial solution $h \in H^{1}(B)$ with $h(1) \neq 0$.

We discuss several applications of Theorem 4.1. The proof of it is postponed to the next section.

As a first corollary of Theorem 4.1, we have

Corollary 4.2. Problem (4.1) does not have any weak radial solution in $H_{0}^{1}(B)$. 
Proof. Suppose on the contrary that there is a weak radial function $k \in H_{0}^{1}(B)$ satisfying

$$
-\Delta k=\left(p u_{*}^{p-1}+\lambda_{*}\right) k .
$$

By the regularity of $-\Delta, k \in C^{1}((0,1])$. Multiplying $k$ on both sides of (4.1) and integrating it on $B$, we obtain that $h(1) k^{\prime}(1)=0$. The maximum principle implies that $k^{\prime}(1)<0$. Then $h(1)=0$, a contradiction.

Next we obtain the following theorem.

Theorem 4.3. Assume that the conditions in Theorem 4.1 hold. Then the Morse index of $u_{*}$ is 1 .

Proof. To see this, we show that for any sequence $\left\{\left(\lambda_{n}, u_{n}\right)\right\} \subset \mathscr{C}$ satisfying $\lambda_{n} \rightarrow$ $\lambda_{*}, \max _{B} u_{n} \rightarrow \infty$,

$$
m\left(u_{n}\right)=1 .
$$

Considering the eigenvalue problem

$$
-\Delta k=\left(p u_{n}^{p-1}+\lambda_{n}\right) k+\kappa k \text { in } B, \quad k=0 \text { on } \partial B,
$$

suppose $m\left(u_{n}\right) \geq 2$. Then the second eigenfunction $k_{n} \in H_{0}^{1}(B) \cap C^{2}(0,1)$ corresponding to the second eigenvalue $\kappa_{n}<0$ of (4.7) satisfies that $k_{n}$ changes sign in $(0,1)$, and we can assume that $k_{n}(0)=\max _{B} k_{n},\left\|k_{n}\right\|_{L^{2}(B)}=1$. We also have that

$$
\lambda_{n}+\kappa_{n}>0 \text { and }\left|\kappa_{n}\right| \leq C
$$

since $\int_{B}\left[\left|\nabla k_{n}\right|^{2}-p u_{n}^{p-1} k_{n}^{2}\right]>0$ and $m\left(u_{n}\right) \leq C$ (see Theorem 3.1). The fact $u_{n} \rightarrow u_{*}$ in $C^{0}(B \backslash\{0\})$ as $n \rightarrow \infty$ and the regularity of $-\Delta$ imply that $\kappa_{n} \rightarrow \tilde{\kappa} \leq 0$, $k_{n} \rightarrow \tilde{k}$ in $C^{2}(B \backslash\{0\})$ as $n \rightarrow \infty$ (we can choose subsequences if necessary) and $\tilde{k} \in H_{0}^{1}(B) \cap C^{2}(B \backslash\{0\})$, which changes sign in $(0,1)$, and satisfies

$$
-\Delta \tilde{k}=\left(p u_{*}^{p-1}+\lambda_{*}\right) \tilde{k}+\tilde{\kappa} \tilde{k} \text { in } B, \quad \tilde{k}=0 \text { on } \partial B .
$$

(Note that $\int_{B}\left|\nabla k_{n}\right|^{2} \leq p \beta+\lambda_{n}$.) We easily derive that $\tilde{\kappa}<0$; otherwise, $\tilde{\kappa}=0$, which contradicts Corollary 4.2 .

The Emden-Fowler transformation as above implies that $\hat{k}(t)=r^{2 /(p-1)} \tilde{k}(r)$ satisfies the problem

$$
\hat{k}^{\prime \prime}+\alpha \hat{k}^{\prime}+p v_{*}^{p-1} \hat{k}-\beta \hat{k}+\left(\lambda_{*}+\tilde{\kappa}\right) e^{2 t} \hat{k}=0 \quad t \in(-\infty, 0), \quad \hat{k}(0)=0 .
$$

Moreover, $\hat{k}$ changes sign in $(-\infty, 0)$. By modifying the proof of Theorem 4.1 (note that $\tilde{\kappa} y(t)>0$ for $t \leq t_{*}$ in (5.8) $)$, we obtain that the fundamental solution $\hat{k}_{*}$ satisfying $\hat{k}_{*}(t) \sim e^{\xi_{1} t}$ at $t=-\infty$ has the property $\hat{k}_{*}(0)<0$. Thus, Corollary 4.2 implies that $\hat{k}$ cannot exist. This completes the proof.

Corollary 4.4. Under the conditions of Theorem 4.3, (1.1) has no regular solution for $\lambda \leq \lambda_{*}$.

Proof. We first consider the case $\lambda<\lambda_{*}$. Arguing by contradiction, we see that there are two possibilities: (i) $u_{*}>u_{\lambda}$ in $\left[0,1\right.$ ), (ii) the graph of $u_{\lambda}$ intersects with that of $u_{*}$ more than two times in $(0,1)$.

Indeed, if the graph of $u_{\lambda}$ intersects with that of $u_{*}$ just once in $(0,1)$, we can easily see that $\lambda>\lambda_{*}$. Making the changes

$$
t=\lambda_{*}^{1 / 2} r, \quad w_{*}(t)=\lambda_{*}^{-1 /(p-1)} u_{*}(r)
$$


and

$$
t=\lambda^{1 / 2} r, \quad w_{\lambda}(t)=\lambda^{-1 /(p-1)} u_{\lambda}(r),
$$

we see that $w_{*}(t)$ and $w_{\lambda}(t)$ satisfy the problems

$$
\begin{array}{ll}
-\Delta w_{*}=w_{*}^{p}+w_{*}, & w_{*}\left(\lambda_{*}^{1 / 2}\right)=0, \\
-\Delta w_{\lambda}=w_{\lambda}^{p}+w_{\lambda}, & w_{\lambda}\left(\lambda^{1 / 2}\right)=0,
\end{array}
$$

respectively. Since there is $r_{0} \in(0,1)$ such that $u_{*}\left(r_{0}\right)=u_{\lambda}\left(r_{0}\right)$, then $u_{\lambda}(r)>u_{*}(r)$ for $r \in\left(r_{0}, 1\right)$. This implies $w_{\lambda}(t)>w_{*}(t)$ for $t \in\left(\lambda^{1 / 2} r_{0}, \lambda^{1 / 2}\right)$. It is clear that $\lambda_{*}^{1 / 2}>\lambda_{*}^{1 / 2} r_{0}>\lambda^{1 / 2} r_{0}$. Since $w_{*}\left(\lambda_{*}^{1 / 2}\right)=0$ and $w_{\lambda}\left(\lambda^{1 / 2}\right)=0$, we conclude that $\lambda_{*}^{1 / 2}<\lambda^{1 / 2}$. This contradicts the fact that $\lambda<\lambda_{*}$.

Now we show that (i) and (ii) are also impossible.

For case (i), we have that

$$
-\Delta u_{*}=u_{*}^{p}+\lambda_{*} u_{*} \text { in } B, \quad u_{*}=0 \text { on } \partial B
$$

and

$$
-\Delta u_{\lambda}=u_{\lambda}^{p}+\lambda u_{\lambda} \text { in } B, \quad u_{\lambda}=0 \text { on } \partial B .
$$

Multiplying $u_{\lambda}$ on both sides of the equation of $u_{*}$ and integrating it on $B$, we obtain

a contradiction.

$$
\int_{B}\left(u_{*}^{p-1}-u_{\lambda}^{p-1}\right) u_{*} u_{\lambda}=\left(\lambda-\lambda_{*}\right) \int_{B} u_{*} u_{\lambda}<0
$$

For case (ii), we can obtain that $m\left(u_{*}\right) \geq 2$. Indeed, there are $r_{0}<r_{1}<r_{2} \leq 1$ such that $u_{*}>u_{\lambda}$ in $\left(0, r_{0}\right), u_{*}<u_{\lambda}$ in $\left(r_{0}, r_{1}\right)$, and $u_{*}>u_{\lambda}$ in $\left(r_{1}, r_{2}\right)$. Making the changes

$$
t=\lambda_{*}^{1 / 2} r, \quad w_{*}(t)=\lambda_{*}^{-1 /(p-1)} u_{*}(r)
$$

and

$$
t=\lambda^{1 / 2} r, \quad w_{\lambda}(t)=\lambda^{-1 /(p-1)} u_{\lambda}(r),
$$

we see that $w_{*}$ and $w_{\lambda}$ satisfy the problem

$$
\left\{\begin{array}{l}
-w_{*}^{\prime \prime}-\frac{N-1}{t} w_{*}^{\prime}=w_{*}^{p}+w_{*} \text { in }\left(0, \lambda_{*}^{1 / 2}\right), \\
w_{*}>0 \text { in }\left(0, \lambda_{*}^{1 / 2}\right), \quad w_{*}\left(\lambda_{*}^{1 / 2}\right)=0
\end{array}\right.
$$

and

$$
\left\{\begin{array}{l}
-w_{\lambda}^{\prime \prime}-\frac{N-1}{t} w_{\lambda}^{\prime}=w_{\lambda}^{p}+w_{\lambda} \text { in }\left(0, \lambda^{1 / 2}\right), \\
w_{\lambda}>0 \text { in }\left(0, \lambda^{1 / 2}\right), \quad w_{\lambda}\left(\lambda^{1 / 2}\right)=0,
\end{array}\right.
$$

respectively. Now we claim that the graph of $w_{*}$ intersects with that of $w_{\lambda}$ at least two times in $\left(0, \lambda^{1 / 2}\right)$. Since $u_{*}<u_{\lambda}$ in $\left(r_{0}, r_{1}\right)$ and $\lambda^{-1 /(p-1)} \geq \lambda_{*}^{-1 /(p-1)}$, there is an interval $J \subset\left(0, \lambda^{1 / 2}\right)$ such that $w_{\lambda}>w_{*}$ in $J$. On the other hand, since $w_{*}(0)=\infty$, we see that there is an interval $(0, \tilde{t})$ such that $w_{*}>w_{\lambda}$ in $(0, \tilde{t})$. Moreover, since $w_{\lambda}\left(\lambda^{1 / 2}\right)=0, w_{*}\left(\lambda_{*}^{1 / 2}\right)=0$ and $\lambda_{*}>\lambda$, there is another interval $\left(\hat{t}, \lambda^{1 / 2}\right)$ such that $w_{*}>w_{\lambda}$ in $\left(\hat{t}, \lambda^{1 / 2}\right)$. This implies that our claim is true. Let $w_{\lambda} \equiv 0$ in $\left[\lambda^{1 / 2}, \lambda_{*}^{1 / 2}\right]$. Arguments similar to those in the proof of Proposition 2.1 imply that $m\left(w_{*}\right) \geq 2$. This implies that $m\left(u_{*}\right) \geq 2$ and contradicts the fact that $m\left(u_{*}\right)=1$.

It remains to consider the case $\lambda=\lambda_{*}$. Suppose that there is a regular solution $u^{*}$ for (1.1) with $\lambda=\lambda_{*}$. We derive from arguments as above that its graph intersects with the graph of $u_{*}$ exactly once. Now we show that $m\left(u_{*}\right) \geq 2$. Arguments 
similar to those in the proof of Theorem 4.3 imply that there exist $\zeta<0$ with $\lambda_{*}+\zeta>0$ and $m \in H_{0}^{1}(B)$ with $m(r)>0$ for $r \in(0,1)$ such that

$$
\int_{B}\left[|\nabla m|^{2}-\left(p u_{*}^{p-1}+\lambda_{*}\right) m^{2}\right]=\zeta \int_{B} m^{2} .
$$

Thus, $\zeta$ is the first eigenvalue and $m$ is the first eigenfunction of the problem

$$
-\Delta h=\left(p u_{*}^{p-1}+\lambda_{*}\right) h+\kappa h \quad \text { in } B \quad h=0 \text { on } \partial B .
$$

On the other hand, we see that there is $r_{0} \in(0,1)$ such that $u_{*}(r)>u^{*}(r)$ for $r \in\left(0, r_{0}\right)$ and $u_{*}\left(r_{0}\right)=u^{*}\left(r_{0}\right)$. Let

$$
m_{*}(r)= \begin{cases}u_{*}(r)-u^{*}(r) & \text { for } r \in\left(0, r_{0}\right), \\ 0 & \text { otherwise. }\end{cases}
$$

We obtain that

$$
\int_{B_{r_{0}}}\left[\left|\nabla m_{*}\right|^{2}-\left(p u_{*}^{p-1}+\lambda_{*}\right) m_{*}^{2}\right]<0 .
$$

Thus, $m\left(u_{*}\right) \geq 2$. This contradicts $m\left(u_{*}\right)=1$. This completes the proof.

Corollary 4.5. Under the conditions of Theorem 4.3, the graph of a regular solution $u_{\lambda}$ intersects with that of $u_{*}$ only once in $(0,1)$.

Proof. Under the changes

$$
t=\lambda_{*}^{1 / 2} r, \quad w_{*}(t)=\lambda_{*}^{-1 /(p-1)} u_{*}(r)
$$

and

$$
t=\lambda^{1 / 2} r, \quad w_{\lambda}(t)=\lambda^{-1 /(p-1)} u_{\lambda}(r),
$$

$w_{*}(t)$ and $w_{\lambda}(t)$ satisfy the problems

$$
\begin{array}{ll}
-\Delta w_{*}=w_{*}^{p}+w_{*}, & w_{*}\left(\lambda_{*}^{1 / 2}\right)=0, \\
-\Delta w_{\lambda}=w_{\lambda}^{p}+w_{\lambda}, & w_{\lambda}\left(\lambda^{1 / 2}\right)=0,
\end{array}
$$

respectively. Since $w_{\lambda}(0)<w_{*}(0)$ and $\lambda>\lambda_{*}$, we easily see that the graph of $w_{\lambda}$ intersects with that of $w_{*}$. This implies that the graph of $u_{\lambda}$ must intersect with that of $u_{*}$. If the graph of $u_{\lambda}$ intersects with that of $u_{*}$ more than two times, we can easily obtain that $m\left(u_{*}\right) \geq 2$. This is a contradiction.

Proof of Theorem 1.3. Theorem 1.3 follows from Theorem 4.3 and Corollaries 4.4 and 4.5 ,

\section{Proof of Theorem 4.1}

This section is devoted to the proof of Theorem 4.1 Recall that $\psi_{1}$ is a solution of (4.2). Our aim is to show that $\psi_{1}$ changes sign only once in $(-\infty, 0)$ and

$$
\psi_{1}(0)<0 \text {. }
$$

We first show that $\psi_{1}$ must change sign in $(-\infty, 0)$. In fact, suppose to the contrary. Since $\psi_{1}>0$ as $t \rightarrow-\infty$, we may assume $\psi_{1}>0$ in $(-\infty, 0)$. Note that $v_{*}$ satisfies

$$
L v_{*}=(p-1) v_{*}^{p},
$$

where

$$
L \psi=\psi^{\prime \prime}+\alpha \psi^{\prime}-\beta \psi+p v_{*}^{p-1} \psi+\lambda_{*} e^{2 t} \psi .
$$


Thus

$$
\begin{aligned}
(p-1) \int_{-\infty}^{0} v_{*}^{p} \psi_{1} e^{\alpha t} & =(p-1) \int_{-\infty}^{0} e^{\alpha t}\left(L v_{*}\right) \psi_{1} \\
& =\left.e^{\alpha t}\left(v_{*}^{\prime} \psi_{1}-v_{*} \psi_{1}^{\prime}\right)\right|_{-\infty} ^{0} \\
& =v_{*}^{\prime}(0) \psi_{1}(0) .
\end{aligned}
$$

This is impossible since $v_{*}^{\prime}(0)<0$.

Let $\tilde{\psi}(t)=e^{\frac{\alpha}{2} t} \psi_{1}(t)$. We see that $\tilde{\psi}(t) \sim e^{\frac{t}{2} \sqrt{\alpha^{2}-4(p-1) \beta}}$. Thus

$$
\tilde{\psi}(-\infty)=0 \text { for } p>p_{c} .
$$

(Note that $\alpha^{2}-4(p-1) \beta>0$ provided $p>p_{c}$.) Moreover, $\tilde{\psi}$ satisfies the problem

$$
\tilde{\psi}^{\prime \prime}-\left(\beta+\frac{\alpha^{2}}{4}\right) \tilde{\psi}+p v_{*}^{p-1} \tilde{\psi}+\lambda_{*} e^{2 t} \tilde{\psi}=0, \quad \tilde{\psi}(-\infty)=0 .
$$

Let $J_{\nu}(r)$ denote the Bessel function satisfying (1.6). We denote the first two zeroes of $J_{\nu}(r)$ by $j_{1, \nu}$ and $j_{2, \nu}$. Under the Emden-Fowler transformations

$$
\psi_{\nu}(t)=J_{\nu}(r), \quad t=\ln r
$$

we see that

$$
\psi_{\nu}^{\prime \prime}(t)-\nu^{2} \psi_{\nu}+e^{2 t} \psi_{\nu}=0, \quad t \in(-\infty, \infty)
$$

and the first and second zeroes of $\psi_{\nu}(t)$ are $\ln j_{1, \nu}$ and $\ln j_{2, \nu}$.

Let $\rho=t-\ln j_{i, \nu}(i=1,2)$ and $\varphi_{\nu}(\rho)=\psi_{\nu}(t)$. From [14] we deduce that for $\nu>0$, the first and the second eigenvalues of the problem

$$
\varphi_{\nu}^{\prime \prime}-\nu^{2} \varphi_{\nu}+\eta e^{2 \rho} \varphi_{\nu}=0, \quad \varphi_{\nu}(0)=0
$$

have the following asymptotic expansions:

$$
\begin{aligned}
& \eta_{1, \nu}=\left(j_{1, \nu}\right)^{2}=\nu^{2}-2 \tilde{a}_{1} \nu^{4 / 3}+O\left(\nu^{2 / 3}\right), \\
& \eta_{2, \nu}=\left(j_{1, \nu}\right)^{2}=\nu^{2}-2 \tilde{a}_{2} \nu^{4 / 3}+O\left(\nu^{2 / 3}\right),
\end{aligned}
$$

where $\tilde{a}_{1}>\tilde{a}_{2}$. The corresponding eigenfunctions are

$$
\begin{array}{ll}
\varphi_{1}(\rho)=\psi_{\nu}\left(\rho+\ln j_{1, \nu}\right), & \rho \in(-\infty, 0), \\
\varphi_{2}(\rho)=\psi_{\nu}\left(\rho+\ln j_{2, \nu}\right), & \rho \in(-\infty, 0) .
\end{array}
$$

Let $w_{0}(t)=A(p, N)\left(1-\theta e^{2 t}\right)$ with some $0<\theta<B(p, N)$, where $B(p, N)$ is given in (1.2) (we will choose $\theta$ below). Let $v_{*}(t)=w_{0}(t)+y(t)$. Then,

$$
v_{*}^{p}=\left(w_{0}(t)+y(t)\right)^{p} \geq A(p, N)^{p}\left(1-p \theta e^{2 t}\right)+p w_{0}^{p-1} y(t) .
$$

Moreover, $y$ satisfies the equation

$$
\begin{aligned}
y^{\prime \prime}+\alpha y^{\prime}-\beta y+p w_{0}^{p-1} y+\lambda_{*} e^{2 t} y+ & \lambda_{*} e^{2 t} A(p, N)\left(1-\theta e^{2 t}\right) \\
& -\theta[4+2 \alpha+(p-1) \beta] A(p, N) e^{2 t} \leq 0 .
\end{aligned}
$$

We now choose $t_{*}$ such that

$$
\lambda_{*}\left(1-\theta e^{2 t_{*}}\right)-\theta\left(\lambda_{*}+4+2 \alpha+(p-1) \beta\right)=0 .
$$

Observe that for $t \leq t_{*}$,

$$
\lambda_{*}\left(1-\theta e^{2 t}\right)-\theta\left(\lambda_{*}+4+2 \alpha+\beta(p-1)\right) \geq 0 .
$$


Hence $y$ satisfies the equation

$$
y^{\prime \prime}+\alpha y^{\prime}-\beta y+p w_{0}^{p-1} y+\lambda_{*} e^{2 t} y \leq 0, \quad t \leq t_{*} .
$$

On the other hand, for any $p$, we have

$$
p w_{0}^{p-1} \leq p \beta\left[1-\min \{p-1,1\} \theta e^{2 t}\right] .
$$

Let $\tilde{y}(t)=e^{\frac{\alpha}{2} t} y(t)$. Then $\tilde{y}(t)$ satisfies

$$
\tilde{y}^{\prime \prime}(t)-\left(\frac{\alpha^{2}}{4}-\beta(p-1)\right) \tilde{y}+\left(\lambda_{*}+p w_{0}^{p-1}-p \beta\right) \leq 0 .
$$

Note that

$$
\tilde{y}(t)<0, \quad \tilde{y}^{\prime}(t)<0 \text { for } t \text { near }-\infty
$$

and

$$
\lambda_{*} e^{2 t}+p w_{0}^{p-1}-p \beta \leq\left(\lambda_{*}-p \beta \min \{p-1,1\} \theta\right) e^{2 t} .
$$

Since the first zero of $\psi_{\nu}(t)$ is $\ln j_{1, \nu}$, if we set

$$
t=\ln j_{1, \nu}+s, \quad \hat{\psi}_{\nu}(s)=\psi_{\nu}(t)
$$

we see that $\hat{\psi}_{\nu}(s)$ satisfies

$$
\hat{\psi}_{\nu}^{\prime \prime}-\nu^{2} \hat{\psi}_{\nu}+j_{1, \nu}^{2} e^{2 s} \hat{\psi}_{\nu}=0, \quad s<0, \quad \hat{\psi}_{\nu}(0)=0 .
$$

Therefore, to keep $\tilde{y}(t)<0$ for $t \in\left(-\infty, t_{*}\right)$, we only need to have

$$
\left(\lambda_{*}-p \min \{p-1,1\} \beta \theta\right) e^{2 t_{*}} \leq j_{1, \nu}^{2},
$$

with $\nu^{2}=\frac{\alpha^{2}}{4}-(p-1) \beta$. Indeed, setting

$$
\hat{y}(s)=\tilde{y}(t), \quad t=t_{*}+s,
$$

we see from (5.11) that

$$
\hat{y}^{\prime \prime}-\left(\frac{\alpha^{2}}{4}-\beta(p-1)\right) \hat{y}+\Theta(s) \hat{y} \leq 0, \quad s<0,
$$

where

$$
\Theta(s)=\left(\lambda_{*}-p \beta \min \{p-1,1\} \theta\right) e^{2 t_{*}} e^{2 s} .
$$

It follows from (5.13) and (5.16) that if

$$
\left(\lambda_{*}-p \beta \min \{p-1,1\} \theta\right) e^{2 t_{*}} \leq j_{1, \nu}^{2},
$$

then

This implies

$$
\hat{y}(s)<0 \text { for } s<0 \text {. }
$$

Thus, we obtain another estimate for $t_{*}$,

$$
e^{2 t_{*}} \leq \frac{j_{1, \nu}^{2}}{\lambda_{*}-p \min \{p-1,1\} \beta \theta} .
$$

Combining (5.9) and (5.17), we need

$$
e^{2 t_{*}} \leq \min \left(\frac{1}{\theta}-\frac{4+2 \alpha+(p-1) \beta}{\lambda_{*}}, \frac{j_{1, \nu}^{2}}{\lambda_{*}-p \min \{p-1,1\} \beta \theta}\right) .
$$

Now we choose

$$
\frac{1}{\theta}-\frac{4+2 \alpha+(p-1) \beta}{\lambda_{*}}=\frac{j_{1, \nu}^{2}}{\lambda_{*}-p \min \{p-1,1\} \beta \theta} .
$$


Then, for $p \geq 2$,

$$
\theta=\frac{\lambda_{*}}{p \beta} \tilde{\theta}
$$

where

$$
\tilde{\theta}=\frac{Q+p \beta+j_{1, \nu}^{2}-\sqrt{\left(Q+p \beta+j_{1, \nu}^{2}\right)^{2}-4 p \beta Q}}{2 Q}
$$

and

$$
Q=4+2 \alpha+(p-1) \beta=4\left(N-1-\frac{3}{p-1}\right)=\frac{16 \lambda_{*}}{B(p, N)} .
$$

Since $\left(Q+p \beta+j_{1, \nu}^{2}\right)^{2}-4 p \beta Q>\left(Q-p \beta+j_{1, \nu}^{2}\right)^{2}$, we have that

$$
\tilde{\theta}<\frac{p \beta}{Q}=\frac{p \beta B(p, N)}{16 \lambda_{*}} .
$$

Therefore,

$$
\theta<\frac{B(p, N)}{16}
$$

Thus,

$$
e^{2 t_{*}}=\frac{p \beta-Q \tilde{\theta}}{\lambda_{*} \tilde{\theta}} .
$$

By the above arguments, we deduce that the solution $\tilde{\psi}(t)$ of (5.3) has no zero in $\left(-\infty, t_{*}\right)$. Indeed, since $v_{*}^{p-1}<w_{0}^{p-1}$ in $\left(-\infty, t_{*}\right)$, under the change $t=t_{*}+s$, we have

$$
\begin{aligned}
p v_{*}^{p-1}\left(t_{*}+s\right)+\lambda_{*} e^{2 t_{*}} e^{2 s} & <p w_{0}^{p-1}\left(t_{*}+s\right)+\lambda_{*} e^{2 t_{*}} e^{2 s} \\
& \leq p \beta+\left[\lambda_{*}-\min \{p-1,1\} p \beta \theta\right] e^{2 t_{*}} e^{2 s} \\
& \leq p \beta+j_{1, \nu}^{2} e^{2 s} .
\end{aligned}
$$

The Sturm's comparison principle implies that $\tilde{\psi}$ has no zero in $\left(-\infty, t_{*}\right)$, as otherwise, $\hat{\psi}_{\nu}$ (with $\nu^{2}=\frac{\alpha^{2}}{4}-(p-1) \beta$ ) has a zero in $(-\infty, 0)$, which is impossible.

On the other hand, we have that

$$
v_{*}\left(t_{*}\right) \leq w_{0}\left(t_{*}\right)=\frac{Q \tilde{\theta}}{p \beta} \beta^{1 /(p-1)} .
$$

Since $u_{*}^{\prime}(r)<0$ for $r \in(0,1), e^{-\frac{2}{p-1} t} v_{*}(t)$ is decreasing in $(-\infty, 0)$. Thus

$$
e^{-\frac{2}{p-1} t} v_{*}(t) \leq e^{-\frac{2}{p-1} t_{*}} v_{*}\left(t_{*}\right) \text { for } t \in\left[t_{*}, 0\right) \text {. }
$$

The fact that

$$
e^{-\frac{2}{p-1} t_{*}}=\left[\frac{\lambda_{*} \tilde{\theta}}{p \beta-Q \tilde{\theta}}\right]^{1 /(p-1)}
$$

and (5.22) imply that for $t \in\left[t_{*}, 0\right)$,

$$
p v_{*}^{p-1}(t) e^{-2 t} \leq\left(\frac{Q \tilde{\theta}}{p \beta}\right)^{p-1} \frac{\lambda_{*} \tilde{\theta}}{p \beta-Q \tilde{\theta}} p \beta .
$$

Since

$$
\left(Q+p \beta+j_{1, \nu}^{2}\right)^{2}-4 p \beta Q=\left(Q-p \beta+j_{1, \nu}^{2}\right)^{2}+4 p \beta j_{1, \nu}^{2}
$$


and

$$
\frac{4 p \beta j_{1, \nu}^{2}}{\left(Q-p \beta+j_{1, \nu}^{2}\right)^{2}} \text { is bounded for any } p
$$

(note that $1 \leq p \beta \leq(N-2)^{2} / 4$ for $p \geq p_{c}$ ), we obtain that

$$
\begin{aligned}
p \beta-Q \tilde{\theta} & =\frac{1}{2}\left[-\left(Q-p \beta+j_{1, \nu}^{2}\right)+\left|Q-p \beta+j_{1, \nu}^{2}\right| \sqrt{\left.1+\frac{4 p \beta j_{1, \nu}^{2}}{\left(Q-p \beta+j_{1, \nu}^{2}\right)^{2}}\right]}\right. \\
& >\tau \frac{p \beta j_{1, \nu}^{2}}{Q-p \beta+j_{1, \nu}^{2}}
\end{aligned}
$$

with $0<\tau<1$ independent of $p$, provided

$$
Q-p \beta+j_{1, \nu}^{2}>0
$$

(Note that $(1+x)^{1 / 2}=1+\frac{1}{2}(1+\xi)^{-1 / 2} x \geq 1+\frac{1}{2}(1+x)^{-1 / 2} x$.) The case $Q-p \beta+$ $j_{1, \nu}^{2} \leq 0$ can be treated similarly. Indeed, if $Q-p \beta+j_{1, \nu}^{2}=0$, then

$$
p \beta=Q \tilde{\theta}+\sqrt{p \beta j_{1, \nu}^{2}} .
$$

Thus,

If $Q-p \beta+j_{1, \nu}^{2}<0$, we obtain that

$$
\frac{Q \tilde{\theta}}{p \beta}=1-\frac{\sqrt{p \beta j_{1, \nu}^{2}}}{p \beta}:=\tilde{\tau}<1 .
$$

$$
p \beta-Q \tilde{\theta}>\tau \frac{p \beta j_{1, \nu}^{2}}{\left|Q-p \beta+j_{1, \nu}^{2}\right|} .
$$

We can also choose $\tilde{\tau}$ similarly. Therefore, without loss of generality, we only consider the case $Q-p \beta+j_{1, \nu}^{2}>0$. Thus

$$
\frac{Q \tilde{\theta}}{p \beta}<\frac{Q-p \beta+(1-\tau) j_{1, \nu}^{2}}{Q-p \beta+j_{1, \nu}^{2}}:=\tilde{\tau}<1,
$$

where $\tilde{\tau}$ is independent of $p$. Therefore, it follows from (5.23) that

$$
\begin{aligned}
p v_{*}^{p-1}(t) e^{-2 t} & \leq \frac{\mu_{1} p \beta\left(Q-p \beta+(1-\tau) j_{1, \nu}^{2}\right)}{Q \tau j_{1, \nu}^{2}} \tilde{\tau}^{p-1} \\
& \leq \frac{\mu_{1}(N-2)^{2}\left(Q-1+(1-\tau) j_{1, \nu}^{2}\right)}{4 Q \tau j_{1, \nu}^{2}} \tilde{\tau}^{p-1} .
\end{aligned}
$$

Now, we conclude that when

$$
\begin{gathered}
p \geq 1+\frac{\ln \frac{4\left(\mu_{1}-\lambda_{*}\right) Q \tau j_{1, \nu}^{2}}{\mu_{1}(N-2)^{2}\left(Q-1+(1-\tau) j_{1, \nu}^{2}\right)}}{\ln \tilde{\tau}}, \\
p v_{*}^{p-1}(t) e^{-2 t} \leq \mu_{1}-\lambda_{*} \text { for } t \in\left[t_{*}, 0\right),
\end{gathered}
$$

where $\mu_{1}$ is the first eigenvalue of the problem

$$
-\Delta v=\mu v \text { in } B, \quad v=0 \text { on } \partial B .
$$

Let

$$
P:=P(N)=1+\frac{\ln \frac{4\left(\mu_{1}-\lambda_{*}\right) Q \tau j_{1, \nu}^{2}}{\mu_{1}(N-2)^{2}\left(Q-1+(1-\tau) j_{1, \nu}^{2}\right)}}{\ln \tilde{\tau}} .
$$


Then,

$$
p v_{*}^{p-1}(t) e^{-2 t}+\lambda_{*} \leq \mu_{1} \text { for } t \in\left[t_{*}, 0\right),
$$

provided $p \geq \max \left\{p_{c}, P, 2\right\}$.

On the other hand, we note that the first eigenfunction $q_{1}(t)$ corresponding to $\mu_{1}$ satisfies the problem

$$
q_{1}^{\prime \prime}-\frac{(N-2)^{2}}{4} q_{1}+\mu_{1} e^{2 t} q_{1}=0 \text { in }(-\infty, 0), \quad q_{1}(-\infty)=q_{1}(0)=0 .
$$

Moreover, $q_{1}$ does not change sign in $(-\infty, 0)$. It follows from (5.3) that $\tilde{\psi}(t)$ satisfies the problem

$$
\tilde{\psi}^{\prime \prime}(t)-\frac{(N-2)^{2}}{4} \tilde{\psi}+\left(p v_{*}^{p-1} e^{-2 t}+\lambda_{*}\right) e^{2 t} \tilde{\psi}=0, \quad \tilde{\psi}(-\infty)=0 .
$$

Thus, (5.27) and Sturm's comparison principle imply that $\tilde{\psi}$ cannot have two zeroes in $\left[t_{*}, 0\right]$. Otherwise, $q_{1}$ will have a zero in $\left(t_{*}, 0\right)$, a contradiction. Thus, $\tilde{\psi}$ has only one zero in $(-\infty, 0)$ and $\tilde{\psi}(0)<0$. This implies $\psi_{1}(0)<0$. This completes the proof.

Remark 5.1. Following the same arguments as above, we can also show that for any $\lambda \in\left(\lambda_{*}, \mu_{1}\right)$, there exists $P_{\lambda}$ sufficiently large such that for $p \geq P_{\lambda}, m\left(u_{\lambda}\right)=1$. Note that $P_{\lambda}$ depends on $\left(\mu_{1}-\lambda\right)$, then $P_{\lambda} \rightarrow \infty$ as $\lambda \rightarrow \mu_{1}$.

\section{More estimates on $p_{c}^{2}(N)$}

In this section we provide more conditions to guarantee that $m\left(u_{*}\right)=1$. As seen in the last section, we just need to show that $\psi_{1}(0)<0$.

Proposition 6.1. There exists $P:=P_{1}(N)>0$ such that for $p \geq \max \left\{p_{c}, P, 2\right\}$ and

$$
\left(j_{2, \frac{N-2}{2}}^{2} j_{1, \nu}^{2}-j_{1, \frac{N-2}{2}}^{4}\right)\left(j_{1, \frac{N-2}{2}}^{4}+Q j_{2, \frac{N-2}{2}}^{2}\right)+p \beta j_{1, \frac{N-2}{2}}^{4} j_{2, \frac{N-2}{2}}^{2} \geq 0,
$$

where $j_{k, \xi}$ denotes the $k$ th positive zero of the Bessel function $J_{\xi}(x), \nu=\frac{\alpha^{2}}{4}-(p-$ 1) $\beta$, and $Q=4\left(N-1-\frac{3}{p-1}\right)$, then $\psi_{1}(0)<0$. Thus, $m\left(u_{*}\right)=1$. Moreover, $m\left(u_{\lambda}\right)=1$ for $\lambda_{*}<\lambda<\mu_{1}$.

Proof. Arguments similar to those in the proof of Theorem 4.1 imply that, by choosing

$$
P_{1}(N):=1+\frac{\ln \frac{4\left(\mu_{2}-\mu_{1}\right) Q \tau j_{1, \nu}^{2}}{\mu_{1}(N-2)^{2}\left(Q-1+(1-\tau) j_{1, \nu}^{2}\right)}}{\ln \tilde{\tau}},
$$

where $\mu_{2}$ is the second eigenvalue of the problem

$$
-\Delta v=\mu v \text { in } B, \quad v=0 \text { on } \partial B
$$

we see that for $p \geq \max \left\{p_{c}, 2, P_{1}(N)\right\}$,

$$
p v_{*}^{p-1}(t) e^{-2 t} \leq \mu_{2}-\mu_{1}<\mu_{2}-\lambda_{*} \text { for } t \in\left[t_{*}, 0\right) .
$$

Therefore,

$$
p v_{*}^{p-1}(t) e^{-2 t}+\lambda_{*} \leq \mu_{2} \text { for } t \in\left[t_{*}, 0\right),
$$

provided $p \geq \max \left\{p_{c}, P_{1}(N), 2\right\}$. 
On the other hand, we observe that the second eigenfunction $q_{2}(t)$ corresponding to $\mu_{2}$ satisfies the problem

$$
q_{2}^{\prime \prime}-\frac{(N-2)^{2}}{4} q_{2}+\mu_{2} e^{2 t} q_{2}=0 \text { in }(-\infty, 0), \quad q_{2}(-\infty)=q_{2}(0)=0 .
$$

Moreover, $q_{2}$ changes sign only once in $(-\infty, 0)$, and $q_{2}\left(\ln \left(j_{1,(N-2) / 2} / j_{2,(N-2) / 2}\right)\right)=$ 0 . Now we require

$$
t_{*}>\ln \left(j_{1,(N-2) / 2} / j_{2,(N-2) / 2}\right)
$$

Thus,

$$
e^{2 t_{*}}>e^{2 \ln \left(\frac{j_{1,(N-2) / 2}}{j_{2,(N-2) / 2}}\right)}=\left(\frac{j_{1,(N-2) / 2}}{j_{2,(N-2) / 2}}\right)^{2}
$$

Therefore,

$$
p \beta-Q \tilde{\theta} \geq \vartheta^{2} \mu_{1} \tilde{\theta}
$$

where

$$
\vartheta=\frac{j_{1,(N-2) / 2}}{j_{2,(N-2) / 2}}
$$

Then

$$
2 Q p \beta \geq\left(\vartheta^{2} \mu_{1}+Q\right)\left[Q+p \beta+j_{1, \nu}^{2}-\sqrt{\left(Q+p \beta+j_{1, \nu}^{2}\right)^{2}-4 p \beta Q}\right] .
$$

Therefore,

$$
\vartheta^{4} \mu_{1}^{2}+\vartheta^{2} Q \mu_{1} \leq \vartheta^{2} j_{1, \nu}^{2} \mu_{1}+\vartheta^{2} p \beta \mu_{1}+Q j_{1, \nu}^{2}
$$

That is,

$$
\left(j_{2, \frac{N-2}{2}}^{2} j_{1, \nu}^{2}-j_{1, \frac{N-2}{2}}^{4}\right)\left(j_{1,(N-2) / 2}^{4}+Q j_{2,(N-2) / 2}^{2}\right)+p \beta j_{1, \frac{N-2}{2}}^{4} j_{2, \frac{N-2}{2}}^{2} \geq 0 .
$$

It follows from (5.3) that $\tilde{\psi}(t)$ satisfies the problem

$$
\tilde{\psi}^{\prime \prime}(t)-\frac{(N-2)^{2}}{4} \tilde{\psi}+\left(p v_{*}^{p-1} e^{-2 t}+\lambda_{*}\right) e^{2 t} \tilde{\psi}=0, \quad \tilde{\psi}(-\infty)=0 .
$$

Thus, (6.4), (6.6) and the Sturm comparison principle imply that $\tilde{\psi}$ cannot have two zeroes in $\left[t_{*}, 0\right]$. Otherwise, $q_{2}$ will have two zeros in $(-\infty, 0)$, a contradiction. Thus, $\tilde{\psi}$ has only one zero in $(-\infty, 0)$ and $\tilde{\psi}(0)<0$. This implies $\psi_{1}(0)<0$. Therefore, we have $m\left(u_{*}\right)=1$. Similar arguments imply that $m\left(u_{\lambda}\right)=1$ for $\lambda_{*}<\lambda<\mu_{1}$.

We see from [14] that for $k=1,2$

$$
\mu+\frac{a_{k}}{2^{1 / 3}} \mu^{1 / 3}<j_{k, \mu}<\mu+\frac{a_{k}}{2^{1 / 3}} \mu^{1 / 3}+\frac{3}{20} a_{k}^{2} \frac{2^{1 / 3}}{\mu^{1 / 3}},
$$

where $a_{1}=2.33811$ and $a_{2}=4.08795$. Using Matlab, we find that when $N=12$ and $p \geq 12.154 ; N=13$ and $p \geq 4.858 ; N=14$ and $p \geq 3.313 ; N=15$ and $p \geq 2.644 ; N=16$ and $p \geq 2.27 ; N=17$ and $p \geq 2.032 ; N=18$ and $p \geq 1.868$; $N=19$ and $p \geq 1.747 ; N=20$ and $p \geq 1.655 ; N=30$ and $p \geq 1.2870$, (6.9) holds. When $N=11$, (6.9) holds for very large $p$. Since $\nu^{2}=\frac{\alpha^{2}}{4}-(p-1) \beta=0$ for $p=p_{c}$, our arguments cannot be used to deal with this case. Thus, $m\left(u_{*}\right)=1$ for $N \geq 12$, provided that $p$ is suitably large. This completes the proof.

Another estimate for $p_{c}^{2}$ is given by the following. 
Proposition 6.2. Assume

$$
\mu_{1} \leq j_{2, \nu}^{2}
$$

with $\nu=\frac{\alpha^{2}}{4}-(p-1) \beta$. Then $\psi_{1}(0)<0$. Therefore, $m\left(u_{*}\right)=1$. Moreover, $m\left(u_{\lambda}\right)=1$ for all $\lambda_{*}<\lambda<\mu_{1}$.

Proof. Since

$$
p v_{*}^{p-1}+\lambda_{*} e^{2 t}<p \beta+\mu_{1} e^{2 t}
$$

if $\mu_{1} \leq j_{2, \nu}^{2}$ and $\tilde{\psi}$ has two zeros in $(-\infty, 0]$, we have that the second eigenfunction $\tilde{q}_{2}(t)$ corresponding to the problem

$$
\tilde{q}_{2}^{\prime \prime}(t)-\nu^{2} \tilde{q}_{2}(t)+j_{2, \nu}^{2} e^{2 t} \tilde{q}_{2}(t)=0, \quad \tilde{q}_{2}(-\infty)=\tilde{q}_{2}(0)=0
$$

has two zeros in $(-\infty, 0)$. This is a contradiction. Therefore, $\psi_{1}(0)<0$. This implies $m\left(u_{*}\right)=1$. Since $p u_{\lambda}^{p-1} \leq p \beta$ for $\lambda_{*}<\lambda<\mu_{1}$, we can easily see that $m\left(u_{\lambda}\right)=1$ for any regular solution $u_{\lambda}$, provided that (6.12) holds.

Using Matlab, we find that when $N=12$ and $p \geq 13.451 ; N=13$ and $p \geq$ 5.1532; $N=14$ and $p \geq 3.4708 ; N=15$ and $p \geq 2.7487 ; N=30$ and $p \geq 1.1343$, (6.12) holds. This implies that for $p$ large, $m\left(u_{*}\right)=1$. Since $\nu^{2}=\frac{\alpha^{2}}{4}-(p-1) \beta=0$ for $p=p_{c}$, we see that (6.12) cannot hold for $p=p_{c}$. Thus, our argument cannot be used to deal with the case $p=p_{c}$. Moreover, when $N=11$, 6.12) only holds for very large $p$.

We also have the following condition to guarantee $m\left(u_{*}\right)=1$.

Proposition 6.3. Let $t_{*}$ be as in Theorem 4.1. Then $\psi_{1}(0)<0$ provided that

$$
\left|t_{*}\right| \leq \frac{\pi}{\sqrt{\lambda_{*}-\left(\frac{\alpha^{2}}{4}-\beta(p-1)\right)}} .
$$

Proof. Note that

$$
p v_{*}^{p-1}+\lambda_{*} e^{2 t} \leq p \beta+\lambda_{*} \text { in }(-\infty, 0) .
$$

Suppose that $\tilde{\psi}$ has two zeroes at $\hat{t}_{1}, \hat{t}_{2}$ in $\left(t_{*}, 0\right]$. Then we have

$$
\left|t_{*}\right|>\frac{\pi}{\sqrt{\lambda_{*}-\left(\frac{\alpha^{2}}{4}-\beta(p-1)\right)}} .
$$

On the contrary, we deduce that there is a solution $\ell(t)$ of the problem

$$
\ell^{\prime \prime}-\left[\left(\frac{\alpha^{2}}{4}-\beta(p-1)\right)-\lambda_{*}\right] \ell=0
$$

such that $\ell>0$ in $\left[\hat{t}_{1}, \hat{t}_{2}\right]$. Let $e(t)=\frac{\tilde{\psi}(t)}{\ell(t)}$. Then $e(t)$ satisfies the equation

$$
e^{\prime \prime}(t)+\frac{2 \ell^{\prime}}{\ell} e^{\prime}(t)+\left[\left(p v_{*}^{p-1}+\lambda_{*} e^{2 t}\right)-\left(p \beta+\lambda_{*}\right)\right] e(t)=0
$$

and $e(t)$ has two zeroes at $\hat{t}_{1}, \hat{t}_{2}$. Moreover, $e<0$ in $\left(\hat{t}_{1}, \hat{t}_{2}\right)$. Thus, $e$ has a minimum at some point in $\left(\hat{t}_{1}, \hat{t}_{2}\right)$; this is impossible. This also implies that if

$$
\left|t_{*}\right| \leq \frac{\pi}{\sqrt{\lambda_{*}-\left(\frac{\alpha^{2}}{4}-\beta(p-1)\right)}},
$$


then $\tilde{\psi}$ changes sign only once in $(-\infty, 0)$ and $\tilde{\psi}(0)<0$. Thus, $\psi_{1}(0)<0$. The condition (6.16) is equivalent to

$$
\frac{1}{2} \ln \frac{(1-\tilde{\theta}) \lambda_{*}}{j_{1, \nu}^{2}} \leq \frac{\pi}{\sqrt{\lambda_{*}-\left(\frac{\alpha^{2}}{4}-\beta(p-1)\right)}}
$$

or

$$
\frac{1}{2} \ln \frac{\lambda_{*} \tilde{\theta}}{p \beta-Q \tilde{\theta}} \leq \frac{\pi}{\sqrt{\lambda_{*}-\left(\frac{\alpha^{2}}{4}-\beta(p-1)\right)}} .
$$

We only need to check the following conditions:

$$
\frac{1}{2} \ln \frac{(1-\tilde{\theta}) \mu_{1}}{j_{1, \nu}^{2}} \leq \frac{\pi}{\sqrt{\mu_{1}-\left(\frac{\alpha^{2}}{4}-\beta(p-1)\right)}}
$$

or

$$
\frac{1}{2} \ln \frac{\mu_{1} \tilde{\theta}}{p \beta-Q \tilde{\theta}} \leq \frac{\pi}{\sqrt{\mu_{1}-\left(\frac{\alpha^{2}}{4}-\beta(p-1)\right)}} .
$$

Remark 6.4. It is known from the numerical data that to guarantee $m\left(u_{*}\right)=1, p$ will be suitably large if $N=11$. This implies that the bound obtained in Theorem 4.1 is reasonable. For $N \geq 12$, we only need a suitably large $p$. Our arguments cannot be used to deal with the case $p=p_{c}$. This case is very delicate. It will be interesting to know the Morse index of $u_{*}$ for this case. We also see that the Morse index of all the radial solutions of (1.1) in $\mathscr{C}$ are 1 , provided that our conditions hold.

\section{ACKNOWLEDGMENTS}

The research of the first author was partially supported by NSFC (10871060) and NSF of Henan Province. The research of the second author was supported by an Earmarked Grant from RGC of Hong Kong and a Focused Research Scheme of CUHK. We thank the referee for reading the manuscript carefully and for valuable remarks.

\section{REFERENCES}

[1] H. Brezis and L. Nirenberg, Positive solutions of nonlinear elliptic equations involving critical Sobolev exponents, Comm. Pure Appl. Math. 36 (1983), 437-477. MR709644 (84h:35059)

[2] C. Budd and J. Norbury, Semilinear elliptic equations and supercritical growth, J. Differential Equations 68 (1987), 169-197. MR892022 (88i:35056)

[3] B. Buffoni, E. N. Dancer and J. Toland, The subharmonic bifurcation of Stokes waves, Arch. Rational Mech. Anal. 152 (2000), 241-271. MR.1764946 (2002e:76010b)

[4] E. N. Dancer, Infinitely many turning points for some supercritical problems, Ann. Math. Pura Appl. 178 (2000), 225-233. MR.1849387 (2002g:35077)

[5] M. del Pino, J. Dolbeault and M. Musso, "Bubble-tower" radial solutions in the slightly supercritical Brezis-Nirenberg problem, J. Differential Equations 193 (2003), 280-306. MR1998635 (2004h:35077)

[6] J. Dolbeault and I. Flores, Geometry of phase space and solutions of semilinear elliptic equations in a ball, Trans. Amer. Math. Soc. 359 (2007), 4073-4087. MR2309176 (2008g:35054)

[7] M. Fila, M. Winkler and E. Yanagida, Convergence rate for a parabolic equation with supercritical nonlinearity, J. Dynam. Diff. Eqns. 17 (2005), no. 2, 249-269. MR2157459 (2006c:35131) 
[8] B. Gidas, W. M. Ni and L. Nirenberg, Symmetry and related properties via the maximum principle, Comm. Math. Phys. 68 (1979), 209-243. MR.544879 (80h:35043)

[9] C. Gui, W.-M. Ni and X. Wang, On the stability and instability of positive steady states of a semilinear heat equation in $R^{n}$, Comm. Pure Appl. Math. 45 (1992), 1153-1181. MR 1177480 (93h:35095)

[10] D. D. Joseph and T. S. Lundgren, Quasilinear Dirichlet problems driven by positive sources, Arch. Rational Mech. Anal. 49 (1973), 241-269. MR0340701(49:5452)

[11] F. Merle and L. A. Peletier, Positive solutions of elliptic equations involving supercritical gowth, Proc. R. Soc. Edinburgh 118A (1991), 49-62. MR1113842 (92g:35074)

[12] F. Merle, L. A. Peletier and J. Serrin, A bifurcation problem at a singular limit, Indiana Univ. Math. J. 43 (1994), 585-609. MR.1291530(95j:35024)

[13] P. Polacik and E. Yanagida, A Liouville property and quasiconvergence for a semilinear heat equation, J. Diff. Eqns. 208 (2005), no. 1, 194-214. MR2107299 (2005j:35109)

[14] C. K. Qu and R. Wong, "Best possible" upper and lower bounds for the zeros of the Bessel function $J_{\nu}(x)$, Trans. Amer. Math. Soc. 351 (1999), 2833-2859. MR.1466955 (99j:33006)

[15] P. H. Rabinowitz, Variational methods for nonlinear eigenvalue problems, Indiana Univ. Math. J. 23 (1974), 729-754. MR0333442(48:11767)

[16] X. Wang, On the Cauchy problem for reaction-diffusion equations, Trans. Amer. Math. Soc. 337 (1993), 549-590. MR.1153016 (93h:35106)

[17] L. Zhang, Uniqueness of positive solutions of $\Delta u+u+u^{p}=0$ in a ball, Comm. PDEs 17 (1992), no. 7-8, 1141-1164. MR1179281 (94b:35125)

Department of Mathematics, Henan Normal University, XinXiang, 453007, People's Republic of China

E-mail address: gzm@htu.cn

Department of Mathematics, The Chinese University of Hong Kong, Shatin, Hong KONG

E-mail address: wei@math.cuhk.edu.hk 